

\title{
Optimization and calibration of flavour tagging algorithms from the $\mathrm{LHCb}$ experiment
}

\section{Antonio Falabella*}

INFN, Bologna; Università di Ferrara

E-mail: falabella@fe.infn.it

In what follows the calibration and performances of the flavour tagging algorithms using the decays $B^{+} \rightarrow \mathrm{J} / \psi K^{+}, B^{0} \rightarrow \mathrm{J} / \psi K^{* 0}$ and $B_{s}^{0} \rightarrow D_{s}^{-} \pi^{+}$are reported. The data sample used correspond to $1.0 \mathrm{fb}^{-1}$ of data collected by the LHCb experiment during 2011. The measured effective tagging efficiency is found to be $2.35 \pm 0.06 \%$ for the opposite side tagging algorithms combination, while it is $1.5 \pm 0.4 \%$ for same side kaon tagging algorithm.

14th International Conference on B-Physics at Hadron Machines, April 8-12, 2013

Bologna, Italy

\footnotetext{
* Speaker.
} 


\section{Flavour Tagging}

One of the main goals of the $\mathrm{LHCb}$ experiment is to perform precise measurement of B meson decays, for instance studying $C P$ violation and very rare decays. For these purposes the $\mathrm{LHCb}$ spectrometer is designed to provide a very selective and efficient trigger, excellent track and vertex resolutions, good mass resolution and excellent identification of particles (pions, protons, kaons, muons and electrons) in a wide range of momenta. For $C P$ violation and time dependent asymmetry measurements it is necessary to know whether the reconstructed B meson initially contain a $b$ or a $\bar{b}$ quark (flavour tagging). In LHCb several flavour tagging algorithms are used: same side (SS) and opposite side (OS) algorithms. The same side algorithm infers the flavour of the B using particles from the fragmentation of the $b$ quark that produce the signal $\mathrm{B}$. When signal is a $\mathrm{B}_{\mathrm{s}}\left(\mathrm{B}_{\mathrm{d}}\right)$ a kaon (pion) is produced; in case it is charged, the sign of the charge determines the initial $b$-content of the signal. The opposite side algorithms (OS) on the other hand use particles from the opposite B meson decay.

In LHCb proton-proton collisions produce $b / \bar{b}$ pairs, that hadronize independently giving the signal B meson and an opposite $\mathrm{B}$. The decay products of this second B can be used to assert the flavour of the signal B. OS algorithms use leptons from semileptonic B decays $(\mu / e)$, kaons from the $b \rightarrow c \rightarrow s$ chain and $Q_{v t x}$ (weighted mean of track charges of the secondary inclusive vertex). Each algorithm provide a tagging decision $(d=+1 / 1)$ for the $\mathrm{B}$ containing a $\bar{b}$ or a $b$ quark based on the charge of the tagging particle [1]. The tagging decision can be wrong if the particle selected to tag is not correct. Moreover OS algorithms decision can be wrong due to the oscillation if the opposite B is neutral.

The sensitivity to a $C P$ asymmetry is directly related to the effective tagging efficiency $\varepsilon_{\text {eff }}=$ $\varepsilon_{\text {tag }}(1-2 \omega)^{2}$ which depends on the mistag fraction $\omega$, which is the fraction of tagged events with a wrong decision, and the tagging efficiency $\varepsilon_{t a g}$ which is the fraction of tagged events. The tagging algorithms have been developed using simulated events (MC). Their performance have been optimised on real data with the purpose of maximising $\varepsilon_{e f f}$.

Each algorithm provide also an estimate of the per-event mistag porbability $(\eta)$ based on the output of a neural network that is trained on simulated events to identify the correct flavour of the signal B. The neural network uses as input geometrical and kinematic variables of the tagger particle and of the event as input. Due to differences between data and simulated events the neural network output must be calibrated in data in order to represent a correct mistag probability. The calibrated mistag estimation $\left(\eta_{c}\right)$ can be used as a per-event mistag probability. The use of the per-event value is found to improve effective tagging efficiency with respect to the mean value. Moreover the per-event value can be used in $C P$ measurements to weight each event.

\section{Control channels}

The mistag $\omega$ can be measured on data using flavour-specific control channels, where the signal $b$-quark content is uniquely defined by their final state. The control channel used are $B^{+} \rightarrow$ $\mathrm{J} / \psi K^{+}, B^{0} \rightarrow \mathrm{J} / \psi K^{* 0}, B^{0} \rightarrow D^{*-} \mu^{+} v_{\mu}, B_{s}^{0} \rightarrow D_{s}^{-} \pi^{+}$. For charged channels the mistag can be measured comparing the charge of the signal B with the tag decision. The mistag $\omega$ can than be measured from the number of rightly and wrongly tagged events. For neutral control channels 
the mistag is determined from a fit to the time-dependent mixing asymmetry whose amplitude is proportional a dilution term $D_{\text {tag }}$ :

$$
A_{\text {mix }}^{\text {meas }}(t)=D_{\text {tag }} D_{t} \cos \left(\Delta m_{d / s} t\right) \propto(1-2 \omega) e^{-\frac{1}{2}\left(\Delta m_{d / s} \sigma_{t}\right)^{2}} \cos \left(\Delta m_{d / s} t\right)
$$

For $B_{s}^{0}$ decays channels, because of the large oscillation frequency, the time resolution factor $D_{t}$ is less than one with the typical value of $45 f s$ for the time resolution. Therefore to correctly determine $\omega$ a good knowledge of the time resolution is required.

\section{Optimization and calibration}

The tagging performances were optimized using $1.0 \mathrm{fb}^{-1}$ of data collected by LHCb during 2011 data taking. The aim of the optimization is to tune the cuts used to select the tagging particles in order to maximize the effective tagging efficiency. The opposite side algorithms have been optimized using the decay channel $B^{+} \rightarrow \mathrm{J} / \psi K^{+}$. The optimization of the same side kaon algorithm is performed studying the $B_{s}^{0} \rightarrow D_{s}^{-} \pi^{+}$decay. The optimized tagging performances measured in the different control channels are summarized in the tables 1 and 2 where the average $\varepsilon_{\text {eff }}$ are reported. For the opposite side combination and same side kaon the effective tagging efficiency are $2.35 \pm 0.06 \%$ and $1.5 \pm 0.4 \%$ respectively.

\begin{tabular}{|c|c|c|c|}
\hline \multicolumn{4}{|c|}{$B^{+} \rightarrow J / \psi K^{+}\left(1.0 \mathrm{fb}^{-1}\right.$ LHCb 2011 data $)$} \\
\hline & $\varepsilon_{\text {tag }}(\%)$ & $\omega(\%)$ & $\varepsilon_{\text {eff }}(\%)$ \\
\hline \hline$\mu$ & $5.20 \pm 0.04$ & $30.8 \pm 0.4$ & $0.77 \pm 0.04$ \\
\hline$e$ & $2.46 \pm 0.03$ & $30.9 \pm 0.6$ & $0.36 \pm 0.03$ \\
\hline$K$ & $17.67 \pm 0.08$ & $39.33 \pm 0.24$ & $0.81 \pm 0.04$ \\
\hline$Q_{\mathrm{vtx}}$ & $18.46 \pm 0.08$ & $40.31 \pm 0.24$ & $0.70 \pm 0.04$ \\
\hline$O S$ & $33.2 \pm 0.09$ & $36.7 \pm 0.2$ & $2.35 \pm 0.06$ \\
\hline
\end{tabular}

Table 1: Performances of the OS algorithms obtained with 1.0fb ${ }^{-1}$ collected by LHCb during 2011.

\begin{tabular}{|c|c|c|c|}
\hline \multicolumn{4}{|c|}{$B_{s}^{0} \rightarrow D_{s}^{-} \pi^{+}\left(1.0 \mathrm{fb}^{-1}\right.$ LHCb 2011 data $)$} \\
\hline & $\varepsilon_{\text {tag }}(\%)$ & $\omega(\%)$ & $\varepsilon_{\text {eff }}(\%)$ \\
\hline \hline$S S K$ & $16.3 \pm 0.4$ & $34.8 \pm 2.1$ & $1.5 \pm 0.4$ \\
\hline
\end{tabular}

Table 2: Performances of the SSK algorithm obtained with $1.0 \mathrm{fb}^{-1}$ collected by LHCb during 2011.

The tagging optimization requires the calibration of the mistag probability $(\eta)$ to the measured mistag fraction $(\omega)$. In fact, when more than one tagging decision is available, these are combined according to the corresponding tagging probabilities $\left(\eta_{i}\right)$ to provide a single combined decision and probability. Due to possible correlations among taggers, the combined mistag probability $\eta$ has to be calibrated on data to match the measured mistag fraction. The calibration model used is $\omega(\eta)=p_{0}+p_{1}(\eta-<\eta>)$, where $p_{0}$ and $p_{1}$ are free parameters and $<\eta>$ is the average mistag 
probability. Deviations from $p_{0}=<\eta>$ and $p_{1}=1$ indicate that $\eta$ is not properly calibrated. The calibration is performed for each tagger and than for the combination.

Figure 1 shows the measured mistag fraction $\omega$ as a function of the calibrated mistag probability $\eta_{c}$ for $B^{+} \rightarrow \mathrm{J} / \psi K^{+}$. The $B^{0} \rightarrow \mathrm{J} / \psi K^{* 0}$ is used to cross-check the validity of the calibration (right plot in Fig. 1). In the table 3 the calibration results for $B^{+} \rightarrow \mathrm{J} / \psi K^{+}$and $B_{s}^{0} \rightarrow D_{s}^{-} \pi^{+}$decay


Figure 1: Calibration plots for the $B^{+} \rightarrow \mathrm{J} / \psi K^{+}$channel(left) and for the $B^{0} \rightarrow \mathrm{J} / \psi K^{* 0}$ channel(right).

channels are reported. The errors are statistical and systematic. The results shows that for both channels the mistag is correctly calibrated within errors. The systematic uncertainty in the calibration parameters have been computed taking into account of possible differences of the parameters related to the data taking conditions (run period, magnet polarity), to the initial flavour of the $\mathrm{B}$ signal and to the control channel.

\begin{tabular}{|c|c|c|c|c|}
\hline Algorithm & Channel & $p_{0}$ & $p_{1}$ & $\eta_{c}$ \\
\hline \hline OS[2] & $B^{+} \rightarrow J / \Psi K^{+}$ & $0.392 \pm 0.002 \pm 0.009$ & $1.035 \pm 0.021 \pm 0.012$ & 0.391 \\
\hline $\mathrm{SS}[4]$ & $B_{s}^{0} \rightarrow D_{s}^{-} \pi^{+}$ & $0.349 \pm 0.015 \pm 0.012$ & $1.00 \pm 0.30 \pm 0.02$ & 0.350 \\
\hline
\end{tabular}

Table 3: Calibration results for $B^{+} \rightarrow \mathrm{J} / \psi K^{+}$and $B_{s}^{0} \rightarrow D_{s}^{-} \pi^{+}$channels. Statistical and systematic errors are reported.

\section{Conclusions}

The optimized and calibrated performances of flavour tagging algorithms with $1 \mathrm{fb}^{-1}$ of data collected in 2011 have been reported. For the OS algorithms the $B^{+} \rightarrow \mathrm{J} / \psi K^{+}$decay channel has been used. In this channel the effective tagging efficiency is $2.35 \pm 0.06 \%$. The SS kaon algorithm has been optimized and calibrated on the $B_{s}^{0} \rightarrow D_{s}^{-} \pi^{+}$channel and the effective tagging efficiency is $1.5 \pm 0.4 \%$.

Tagging algorithms have been used in several LHCb published results such as the measurement of the CP-violating phase $\phi_{s}$ in $B_{s}^{0} \rightarrow \mathrm{J} / \psi K^{+} K^{-}$and $B_{s}^{0} \rightarrow \mathrm{J} / \psi \pi^{+} \pi^{-}$decays [5] where the effective tagging efficiency using OS and SS kaon algorithms is $(3.13 \pm 0.12 \pm 0.20) \%$. For the measurement 
of $B_{s}^{0}-\bar{B}_{s}^{0}$ oscillation frequency $\Delta m_{s}$ [6] the OS and SS kaon algorithms have been used and the effective tagging efficiency is $(3.5 \pm 0.5) \%$. The measurement of time dependent CP-violation in $B_{s}^{0} \rightarrow D_{s}^{\mp} K^{ \pm}$[8] used OS algorithms and the effective tagging efficiency is $1.9 \%$. Flavour tagging algorithms has been used also in charmless two-body $B$ decays studies [9] where the effective tagging efficiency with OS algorithms is $2.3 \pm 0.1 \%$.

\section{References}

[1] M. Calvi et al. (LHCb Collaboration), Flavour Tagging Algorithms and Performances in LHCb, [CERN-LHCB-2007-058].

[2] LHCb Collaboration, Opposite-side flavour tagging of B mesons at the LHCb Performance of flavour tagging algorithms optimised for the analysis of $B_{s}^{0} \rightarrow \mathrm{J} / \psi \phi,[\mathrm{LHCB}-\mathrm{CONF}-2012-026]$.

[3] LHCb Collaboration, Opposite-side flavour tagging of B mesons at the LHCb experiment, [ Eur. Phys. J. C (2012) 72:2022], [arXiv:1202.4979]

[4] LHCb Collaboration, Optimization and calibration of the same-side kaon tagging algorithm using $B_{S}$ hadronic decays in 2011 data, [LHCB-CONF-2012-033].

[5] LHCb Collaboration, Measurement of $C P$-violation and the $B_{s}^{0}$ decay width difference with $B_{s}^{0} \rightarrow \mathrm{J} / \psi K^{+} K^{-}$and $B_{s}^{0} \rightarrow \mathrm{J} / \psi \pi^{+} \pi^{-}$decays, [LHCB-PAPER-2013-002], [arXiv:1304.2600]

[6] LHCb Collaboration, Measurement of the $B_{s}^{0}-\overline{B_{s}^{0}}$ oscillation frequency $\Delta m_{s}$ in $B_{s}^{0} \rightarrow D_{s}^{+} \pi^{-}$, [LHCB-PAPER-2013-006-001].

[7] LHCb Collaboration, Measurement of the $B^{0}-\overline{B^{0}}$ oscillation frequency $\Delta m_{d}$ with the decays $B^{0} \rightarrow D \pi^{+}$and $B^{0} \rightarrow \mathrm{J} / \psi K^{* 0}$, [LHCB-PAPER-2012-032], [arXiv:1210.6750].

[8] LHCb Collaboration, Measurement of time-dependent $C P$-violation observables in $B_{s}^{0} \rightarrow D_{s}^{\mp} K^{ \pm}$, [LHCB-CONF-2012-029].

[9] LHCb Collaboration, Measurement of time-dependent CP violation in charmless two-body B decays, [LHCB-CONF-2012-007]. 